\title{
Spreadsheet-Based Program for the Analysis of DNA Methylation
}

BioTechniques 30:110-114 (January 2001)

\section{Ramaswamy Anbazhagan, James G. Herman, Kalyan Enika, and Edward Gabrielson The Johns Hopkins University School of Medicine, Baltimore, MD, USA}

\section{INTRODUCTION}

While the $\mathrm{CpG}$ dinucleotide occurs at less than $10 \%$ of its expected frequency in the human genome, many gene promoter regions have high densities of $\mathrm{CpG}$ sites. These regions are known as $\mathrm{CpG}$ islands, and methylation of cytosines in $\mathrm{CpG}$ islands is a common mechanism for transcriptional inactivation of genes on the inactivated X-chromosome in females and of silenced imprinted alleles on autosomal chromosomes. Furthermore, methylation is increasingly recognized as an important mechanism for inactivation of tumor suppressor genes in neoplasia (reviewed by Baylin et al. in Reference 2 ).

$\mathrm{CpG}$ islands are defined as regions of DNA ranging in size from 0.5 to $2 \mathrm{~kb}$ that have a $\mathrm{C}+\mathrm{G}$ content of greater than $60 \%$ and a ratio of $\mathrm{CpG}$ to $\mathrm{GpC}$ of at least $0.6(3,4)$. Identification of $\mathrm{CpG}$ islands is a critical initial step for studying transcriptional regulation of genes by methylation. The status of $\mathrm{CpG}$ islands can then be evaluated in the laboratory using restriction enzymes that are methylation specific, by sequencing bisulfite modified DNA, or by methylation-specific PCR (MSP) (5) of bisulfite-modified DNA. Here, we report the adaptation of a commonly available spreadsheet program, Microsoft ${ }^{\circledR}$ Excel $^{\circledR} 2000$, as a tool for identifying $\mathrm{CpG}$ islands and assisting in the analysis of DNA methylation.

\section{MATERIALS AND METHODS}

\section{Downloading and Opening the Program}

We have created an Excel file with a built-in program that performs all major functions required for the analysis of $\mathrm{CpG}$ islands. The program works with Excel 2000. The program file (CpGWin) can be downloaded from the Software Library on the Internet at http:// 128.20.85.49/genomics. After downloading the file, the program can be started in two ways: $(i)$ either open the downloaded file or (ii) start the Excel program and open the downloaded file from within the program using File/ Open. A dialogue box appears, asking to enable or disable macros. Click on the Enable Macros button. When the program opens, you will notice that a new menu named Analyze-CpG is added to the program menu bar. This menu has seven menu items, namely New-Workbook, Format, Count-CpG, Mark-CpG, Bis-Modify, and MakeGraph. These menu items can be executed to perform various functions necessary for the analysis of $\mathrm{CpG}$ islands within the DNA sequences.

\section{Opening a New Workbook and Entering a Sequence}

The Excel workbook is organized as a collection of sheets, with each sheet's name appearing on a tab at the bottom of the workbook. Navigation between sheets is possible by clicking on the tab at the bottom. Refer to the Microsoft User's Guide for further explanation about the organization and use of Excel workbook and sheets. To use this program, go to the newly added AnalyzeCpG menu and click on the New-Workbook menu item. This command will open a new workbook with four sheets. The program automatically formats the Sheet2 as shown in Figure 1. To analyze a specific DNA sequence, enter the nucleotide sequence in cell A1 of Sheet1. Nucleotides in the sequence 
may be entered in either uppercase or lowercase. Ambiguous nucleotides may be designated as " $n$ ". The cells can hold more than 30000 characters and can therefore accommodate long sequences. These sequences may be copied and pasted from other documents. GenBank ${ }^{\circledR}$ sequences viewed through Internet browsers can also be directly copied and pasted along with numbers and spaces. If you copy and paste sequences from GenBank, you will notice that the sequence occupies multiple rows in Sheet1. After entering or pasting the sequence in cell A1 of Sheet1, go to the Analyze-CpG menu and click on the Format menu item. This command removes all the numbers and spaces from the sequence (if there are any), concatenates the sequences in all the rows, and also transfers the sequence from cell A1 of sheet 1 to cell J2 of Sheet2. The com mand also activates Sheet 2 so that you can see the transferred sequence there.

The program automatically fills up cell J3 of Sheet 2 with a formula that will count and display the total number of nucleotides in cell J2 of Sheet2. When a sequence is transferred from Sheet 1 to cell J2 of Sheet2, the nucleotide count of that sequence is auto- matically displayed in cell J3. The nucleotide count in cell $\mathrm{J} 3$ is also updated automatically whenever any sequence entry is altered in cell $\mathrm{J} 2$.

\section{Analyzing the $\mathrm{CpG}$ and $\mathrm{GpC}$ Distribution in Different Regions of the Sequence}

The next step is to designate which portions of the sequence are to be analyzed for $\mathrm{CpG}$ islands. This is done by entering the starting and ending position numbers of the fragments to be analyzed in column $\mathrm{G}$ and $\mathrm{H}$, respectively, below the labels Start and End. Up to six fragments can be entered in these columns with the starting and ending position numbers of their nucleotides. After entering these values, go to the Analyze$\mathrm{CpG}$ menu and click on the Count-CpG menu item. After a few seconds delay (depending on the length of the sequence), the analyzed data are displayed in columns $\mathrm{J}-\mathrm{X}$ as shown in Figure 2.

Column J (labeled "Length") displays the length (number of nucleotides) of each of the fragments entered in columns $\mathrm{G}$ and $\mathrm{H}$. Column L (labeled "Number of $\mathrm{C}+\mathrm{G}$ ") displays the total number of cytosine and guanine nucleotides within each of the fragments.

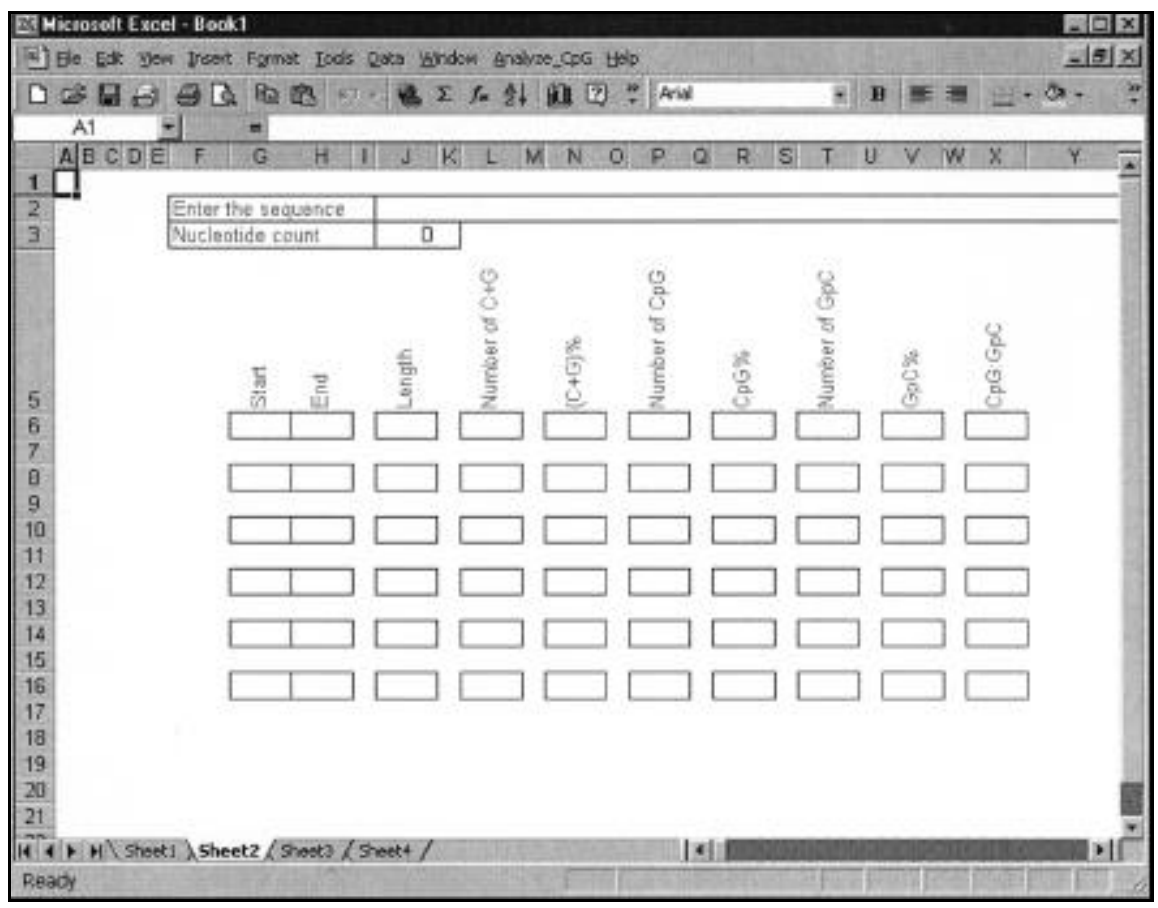

Figure 1. Sheet2 of a new workbook opened by executing the menu item New-Workbook in the CpG-Analyze menu.
The percentage of cytosine and guanine nucleotides within each fragment is displayed in column $\mathrm{N}$ (labeled " $(\mathrm{C}+$ G)\%"). Column P (labeled "Number of $\mathrm{CpG}$ ") displays the total number of $\mathrm{CpG}$ islands within each of these fragments. The percentage of $\mathrm{CpG}$ islands within each of these fragments is displayed in column R (labeled "CpG\%"). Similarly, the total number and the percentage of GpC base pairs are displayed in columns $\mathrm{T}$ and $\mathrm{V}$ (labeled "Number of $\mathrm{GpC}$ " and "GpC\%", respectively). The last column, $\mathrm{X}$, shows the $\mathrm{CpG}: \mathrm{GpC}$ ratio in the sequence.

\section{Marking the CpG and GpC Base Pairs for Visual Display}

Although the various parameters of the $\mathrm{CpG}$ islands are analyzed as described above, it does not give a visual impression of the distribution of the $\mathrm{CpG}$ and $\mathrm{GpC}$ sites within a sequence. The next menu item, Mark-CpG, is meant to perform this function. When this menu item is executed, the program takes the sequence in cell $\mathrm{J} 2$ of Sheet 2 and displays it in columns B and D (labeled "CpG Marked" and "GpC Marked", respectively) of Sheet3 in a vertical column format with each nucleotide occupying a single cell. The program also highlights the $\mathrm{CpG}$ sites in column B and $\mathrm{GpC}$ sites in column $\mathrm{D}$ with a bright pink background. This highlighting helps to visually appreciate the distribution of the $\mathrm{CpG}$ and $\mathrm{GpC}$ sites (Figure 3). This visual effect can be better appreciated by clicking on the View menu bar and executing the Zoom function, which are built-in Excel commands. By adjusting the zoom ratio to $10 \%$ of the original, an overall view the marked sequence can be seen.

\section{Displaying the Bisulfite-Modified DNA Sequence}

MSP and sequencing of bisulfitemodified DNA are commonly employed to analyze methylation of DNA. These techniques both involve treating the DNA with bisulfite, which chemically modifies the unmethylated cytosine residues to uracil. In effect, this modification is reflected as thymine after sequencing or MSP. Bisulfite treatment of DNA therefore results in different prod- 
ucts, depending on the methylation status of the DNA before modification.

The next command in our custom menu, Bis-Modify, helps to identify possible nucleotide sequences after bisulfite treatment, depending on prior methylation status. When this menu item is executed, the DNA sequence from cell $\mathrm{J} 2$ of Sheet 2 is displayed in columns G, H, and I of Sheet3 (labeled "Original", "Unmethylated" and "Methylated", respectively), in a verti-

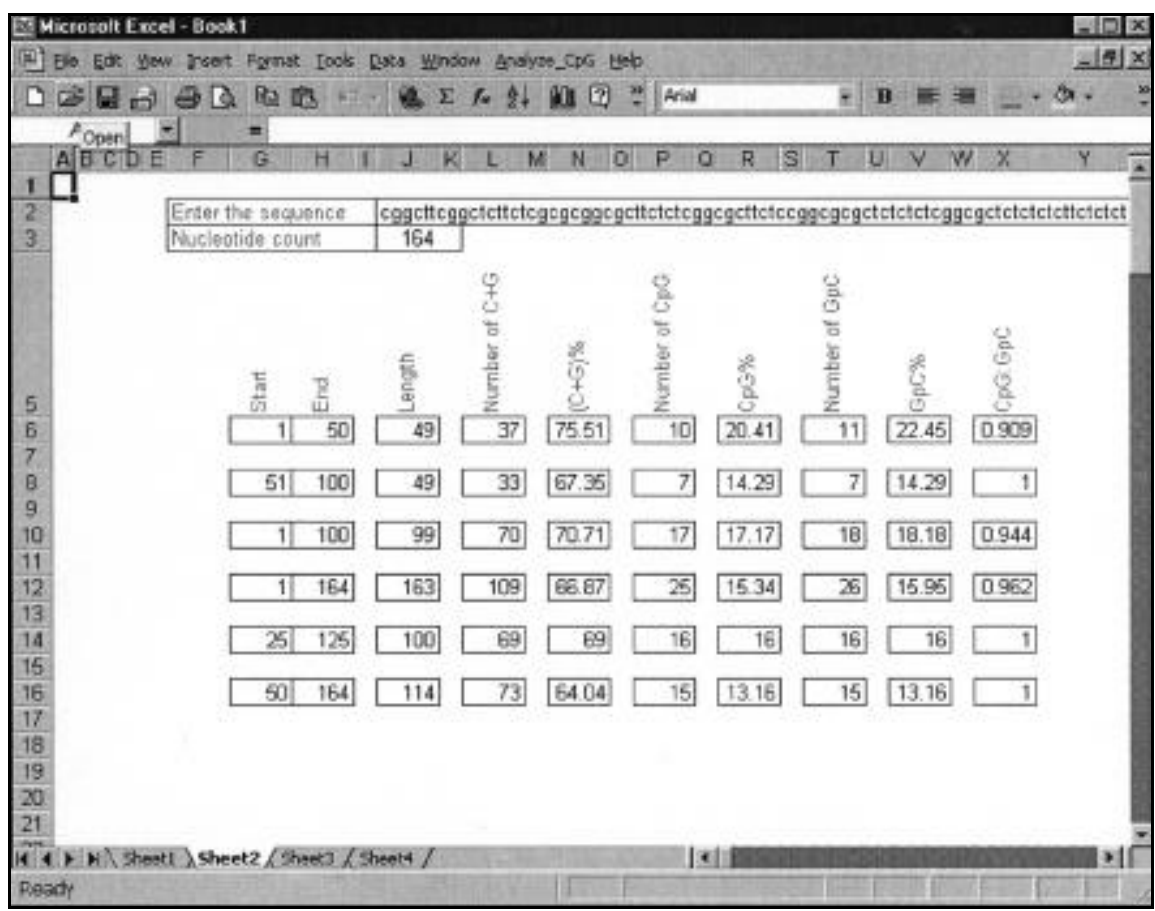

Figure 2. A sample of Sheet 2 displaying the results of analysis of a sequence by executing the Count-CpG menu item.

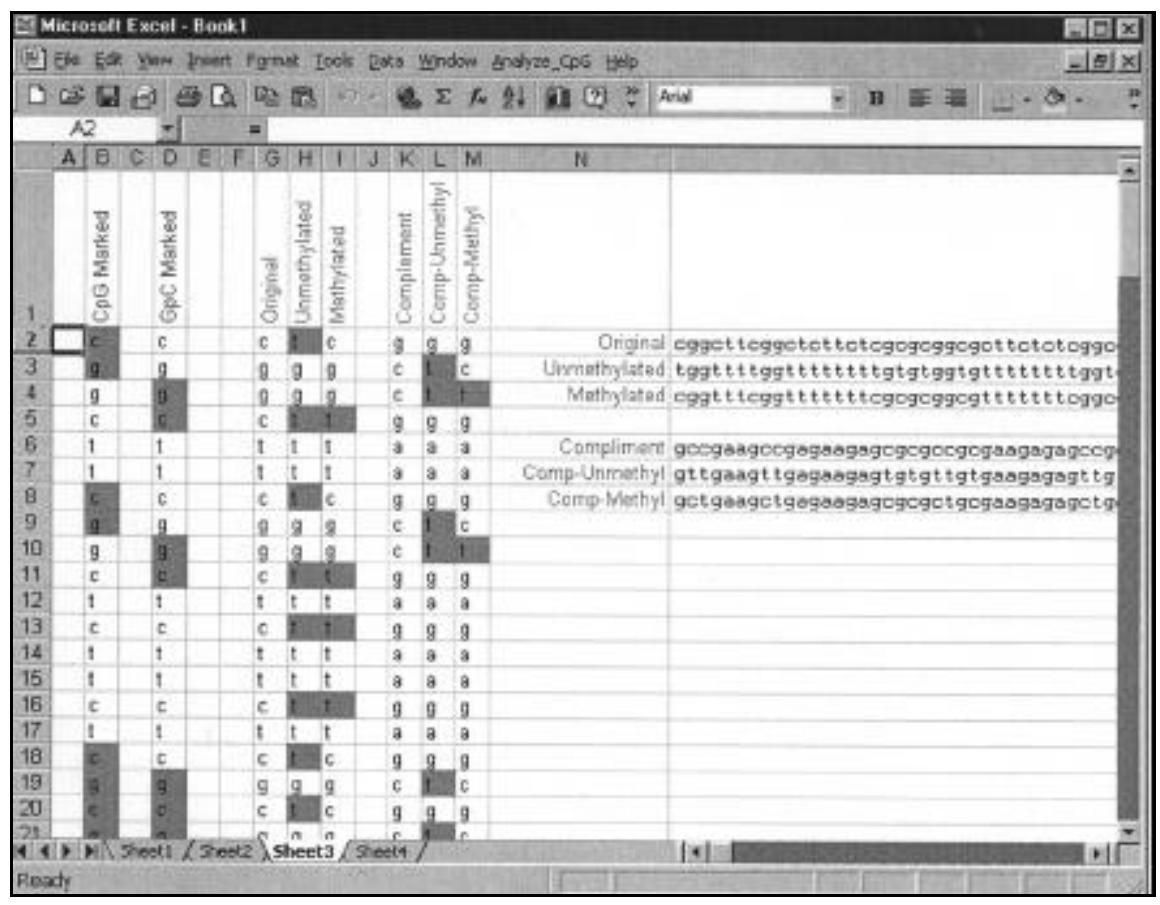

Figure 3. A view of Sheet3 displaying results of executing Mark-CpG and Bis-Modify menu items. cal format, with each nucleotide occupying a single cell. The column labeled "Original" displays the original sequence for the purpose of comparison with others. The column labeled "Unmethylated" displays the modified sequence that would result from bisulfite treatment of unmethylated DNA. Note that in this situation, all cytosine nucleotides would be converted to thymine regardless of whether or not they are associated with a part of a $\mathrm{CpG}$ island. These modified nucleotides are highlighted with a bright pink background. The next column labeled "Methylated" displays the sequence that would result from bisulfite treatment of DNA with methylated sequences in $\mathrm{CpG}$ islands. In this situation, all cytosines not followed by guanine (thus not making a $\mathrm{CpG}$ pair) are modified to thymine, while those followed by guanine (thus making a $\mathrm{CpG}$ pair) are retained as cytosines. As in the previous case, all the modified nucleotides are highlighted with a bright pink background. Thus, the execution of this menu item displays all three sequences (original, unmethylated, and methylated) side by side with the modified nucleotides being highlighted. This display format facilitates comparison of the sequences and identification of possible sites of methylation. The results of bisulfite modification of the complementary strand are displayed in columns $\mathrm{K}, \mathrm{L}$, and $\mathrm{M}$.

To design primers for MSP, it may be desirable to copy these unmodified and modified sequences to another file or to another program used for designing primers. To make it easy to copy these sequences, they are also displayed in a horizontal format with each sequence occupying a single cell as shown in Figure 3. Actually, the menu item Bis-Modify performs this function also. The unmethylated or methylated version of DNA sequence could be easily copied from column $\mathrm{O}$.

\section{Displaying the $\mathrm{CpG}$ and $\mathrm{GpC}$ Base Pairs in a Graphical and Pseudographical Formats}

The next menu item, Make-Graph, is used to display the distribution of $\mathrm{CpG}$ and $\mathrm{GpC}$ base pairs in graphical formats. When this command is execut- 
ed, the program takes the sequence in cell J2 of Sheet2, analyzes the CpG distribution, and plots two graphs. First, the program scans the sequence through a window of $10 \mathrm{bp}$, computes the $\mathrm{CpG}$ frequency, makes a line graph, and displays it in Sheet4 as shown in Figure 4. The $\mathrm{Y}$-axis in this graph represents the exact number of $\mathrm{CpG}$ base pairs counted within a window of 10 bp. Then, the program scans the sequence again through a window of 100 $\mathrm{bp}$, computes the $\mathrm{CpG}$ frequency, and displays the graph in Sheet4 just below the earlier graph. The $\mathrm{Y}$-axis in this graph represents the percentage of $\mathrm{CpG}$ frequency and has a fixed range of $0 \%-12 \%$. While the first graph will be useful for the analysis of shorter fragments, the second will be very useful to analyze larger fragments.

The program also makes a text string "pseudograph" for $\mathrm{CpG}$ and $\mathrm{GpC}$ base pairs. In this pseudograph, the $\mathrm{CpG}$ or $\mathrm{GpC}$ pairs are represented by the "l" symbol, and all other possible pairs of nucleotides are represented by “.” symbol. For example, a sequence such as "acgtcgac" is displayed ".I....." to represent the distribution of the $\mathrm{CpG}$ pairs. These pseudographs are displayed just above the previous graph with labels " $\mathrm{CpG}$ base pairs" and "GpC base pairs". This gives another visual tool to appreciate the distribution of the $\mathrm{CpG}$ islands in a sequence. The sequence and the results can be saved using the built-in Save As command in Excel. The user can then open a new workbook and continue analyzing new sequences, if necessary.

\section{DISCUSSION}

One important feature that we have not included in this program is the ability to search for restriction sites, especially for methylation-specific enzymes within the DNA sequence. We did not include this feature in the current program because our earlier programs, Win-Align and Mac-Align, can perform these functions effectively (1). In summary, we have described a spreadsheet-based program for the analysis of $\mathrm{CpG}$ islands. This program has features to copy and paste sequences from the GenBank database and would be very useful for identifying $\mathrm{CpG}$ frequency in different regions of DNA. The user can get a numerical output of the $\mathrm{CpG}$ frequency in selected regions and appreciate the output visually through a colorshaded vertical display and through graphical and pseudographical formats.

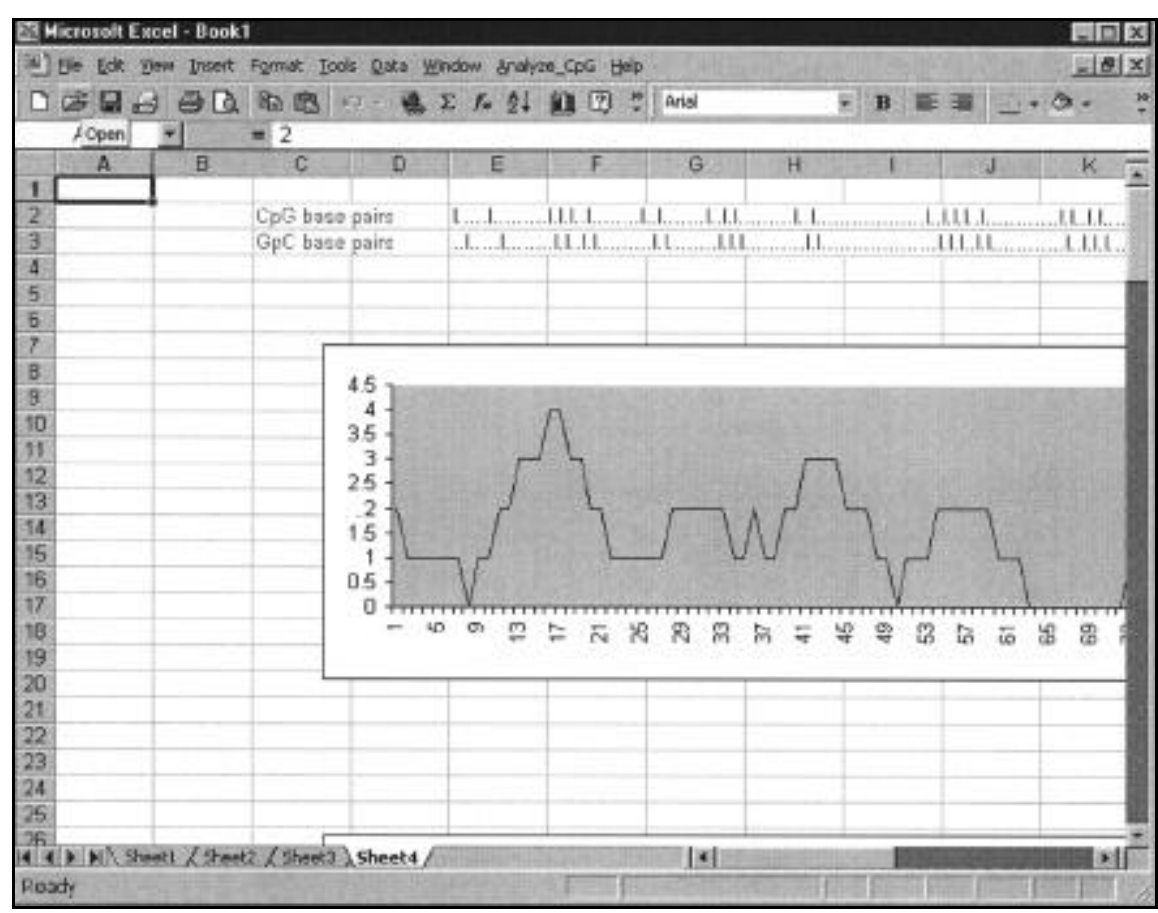

Figure 4. A view of Sheet 3 displaying graphic image and pseudograph of CpG and GpC base pairs.
The program also outputs the sequence that is expected after bisulfite treatment of DNA. This feature will be especially useful for designing primers for methylation-specific PCR.

\section{REFERENCES}

1.Anbazhagan, R. and E. Gabrielson. 1999. Spreadsheet-based program for alignment of overlapping DNA sequences. BioTechniques 26:1180-1185.

2.Baylin, S.B., J.G. Herman, J.R. Graff, P.M. Vertino, and J.P. Issa. 1998. Alterations in DNA methylation: a fundamental aspect of neoplasia. Adv. Cancer. Res. 72:141-196.

3.Bird, A.P. 1986. CpG-rich islands and the function of DNA methylation. Nature 321:209-213.

4.Gardiner-Garden, M. and M. Frommer. 1987. CpG islands in vertebrate genomes. J. Mol. Biol. 196:261-282.

5.Herman, J.G., J.R. Graff, S. Myohanen, B.D. Nelkin, and S.B. Baylin. 1996. Methylation-specific PCR: a novel PCR assay for methylation status of $\mathrm{CpG}$ islands. Proc. Natl. Acad. Sci. USA 93:9821-9826.

Received 10 April 2000; accepted 27 September 2000.

\author{
Address correspondence to: \\ Dr. Ramaswamy Anbazhagan \\ Department of Pathology \\ The Johns Hopkins University School of Medicine \\ Room 301 \\ 418 North Bond Street \\ Baltimore, MD 21231, USA \\ e-mail: anba@jhmi.edu
}

